activity level. This information was used to build an appropriate repeated measures model including demographics and work-related attributes.

Results Final analyses included 318 Latina/o farmworkers. Only 70 subjects (22\%) were a healthy BMI, and 115 subjects $(36 \%)$ were considered obese (BMI $\geq 30)$. None were underweight. The majority of participants were paid hourly $(n=233,73 \%)$ as opposed to piece-rate $(n=85$, 27\%). Activity counts averaged 452, or a low intensity activity level. In the final repeat measures model, work activity was inversely associated with WBGT, for every oC increase in WBGT activity decreased by 4.5 counts (95\% CI 1.2-7.6) P 0.01. Other independent negative associations with activity were found with age and male gender. An interaction was found between piece-rate workers and gender. Male piece rate workers did not decrease activity levels with WBGT, but females and hourly paid workers did.

Conclusion In general, farmworkers decrease their work activity with increasing environmental heat. Men, especially piecerate workers are less likely to decrease their activity levels putting them at increased risk of HRI.

\section{B.2 EPIDEMIOLOGICAL DESCRIPTIONS OF OCCUPATIONAL HEALTH EFFECTS OF CLIMATE CHANGE}

1,2Bruno Lemke*, 2,3 Tord Kjellstrom. ${ }^{1}$ NMIT, Nelson, New Zealand; ${ }^{2}$ CHIRP, Nelson, New Zealand; ${ }^{3} \mathrm{ANU}$, Canberra, Australia

\subsection{6/OEM-2019-EPI.193}

There have been many laboratory studies of the effect of heat on the health of individuals in sport, at work or in the military. However, epidemiological studies are needed to develop impact assessment of climate change. In this presentation we outline the development and use of population-exposure risk functions for different heat effects.

The first risk function is for heat discomfort based on the predicted mean vote with $10 \%$ population feeling discomfort at $\mathrm{WBGT}=21 \mathrm{C}$ and with $90 \%$ affected at $29 \mathrm{C}$.

The second population risk function is for heat exhaustion which we derive by using epidemiological data from the US military. $10 \%$ of the population is affected by heat exhaustion at $\mathrm{WBGT}=31 \mathrm{C}$ increasing up to $90 \%$ of the population affected at a WBGT $=38 \mathrm{C}$.

The most severe population risk is heat stroke for which we use hospital data to calculate $10 \%$ of the population affected by heat stroke at WBGT $=41 \mathrm{C}$ and $90 \%$ of the population at WBGT $=44 \mathrm{C}$.

These health effects of heat create different durations of ill health, with serious heat stroke causing prolonged periods of dis-ability. Based on climate modelling and our risk functions the number of people affected globally can be calculated. For heat stroke, few persons working in the shade at $300 \mathrm{~W}$ metabolic rate, will currently suffer from heat levels that can cause heat stroke. By 2085 half a million workers (at 300W) will be exposed to heat levels that cause heat stroke. As for becoming totally exhausted (and unable to work) while working at $300 \mathrm{~W}$ in the sun, currently that stands at about 124 million worldwide, but in 2085 that number will jump to 835 million.

Additional epidemiological studies are utilised to validate these risk estimates at local and national level.
08B.3 HEAT AND INJURY IN THE WORKPLACE: PERSPECTIVES FROM HEALTH AND SAFETY REPRESENTATIVES

Blesson Varghese*, Alana Hansen, Susan Williams, Peng Bi, Dino Pisaniello. School of Public Health, The University of Adelaide, Adelaide, Australia

\subsection{6/OEM-2019-EPI.194}

Introduction Hot weather poses occupational health and safety concerns for outdoor workers or those in non-cooled indoor environments. The risk of occupational injuries increases during hot weather, however limited understanding exists on underlying factors associated with this increase in risk. While recommendations and guidelines for preventing heat-related health impacts include hydration, cooling practices, adequate ventilation and rescheduling work, the extent to which these recommendations are adopted in workplaces is currently unknown.

Methods A national online survey was conducted among health and safety representatives (HSRs) to better understand the nature of heat-related injuries. Responses relating to risk factors and preventive measures associated with reported injuries in workplaces were identified using log-poisson regression models.

Results In total, 222 HSRs completed the survey. Overall, more than a third $(43 \%)$ of HSRs reported that injuries or incidents caused by hot/very humid weather occur sometimes/ often in their workplace.

Factors found to be positively associated with reported injuries included 'the wearing of personal protective equipment (PPE)', 'inadequate resources and facilities' and 'new workers'.

For outdoor workers, the most frequently adopted preventive measures were provision of PPE, sunscreen and access to cool drinking water. HSRs reported more injuries if certain preventive measures (rescheduling work to cooler times and shaded rest/work areas) were adopted never/rarely/sometimes compared to often/always.

Access to cool drinking water and provision of PPE were the most frequently adopted preventive measures for indoor workers. For this group, HSRs reported more injuries if certain preventive measures (self-pacing, shielding of heat sources and adequate ventilation) were adopted never/rarely/ sometimes.

Conclusion Findings indicate that organisational issues, workplace hazards, personal factors and preventive measures, are all determinants of heat-related injuries in Australian workplaces. Wider adoption of prevention measures such as work rescheduling, self-pacing, provision of shade and adequate ventilation could reduce incidence of heat-related injuries in outdoor and indoor workplaces.

\section{B.4 EXPLORING OCCUPATIONAL INJURY EXPERIENCES DURING HOT WEATHER: A NATIONAL SURVEY OF HEALTH AND SAFETY PROFESSIONALS}

Blesson Varghese*, Dino Pisaniello, Alana Hansen, Susan Williams, Peng Bi. School of Public Health, The University of Adelaide, Adelaide, Australia

\subsection{6/OEM-2019-EPI.195}

Introduction Exposure to extreme heat can lead to adverse health effects and contribute to work-related injuries. However, there is limited understanding of how physical injuries arise in hot weather. A study of the perspectives of 
stakeholders such as occupational health and safety professionals (HSPs) on injury risk factors during hot weather may assist in informing injury prevention efforts.

Methods A national online survey of HSPs was undertaken. Collected data included perspectives on injury experiences, current preventive measures, training, policies and guidelines, and barriers for prevention. Results were analyzed descriptively and a log-poisson regression was used to identify risk factors associated with reported injuries during hot weather at workplaces the HSPs visited/managed.

Results In total there were 307 respondents, the majority of who acknowledged the potential for increased risk of occupational injuries in hot weather. A variety of injury types and mechanisms were reported, including manual handling injuries, hand injuries, wounds or lacerations and loss of control of power tools.

Work factors significantly associated with reported injuries included problems with personal protective equipment; lack of shade; inadequate hydration and rest breaks, and problems with supervision.

Less than half (42\%) of HSPs stated the availability of adequate heat training for staff and 54\% reported the provision for outdoor work to cease if temperatures are extreme as a preventive measure in workplaces they visited/managed. HSPs recommended 'heat stress training' as the most important preventive measure for outdoor and indoor workers. Reported barriers for prevention include: lack of awareness by workers and supervisors of injury risks, and management concerns about productivity loss and/or deadlines.

Conclusion These findings point to a range of modifiable work and organisational risk factors for injury during hot weather. More attention to these factors, in conjunction with traditional interventions for heat-induced illness, could enhance injury prevention in the workplace.

\section{B.5 OCCUPATIONAL HEAT EXPOSURE AND CARDIOVASCULAR HEALTH RISKS RELATED TO CLIMATE CHANGE IN PACIFIC COUNTRIES}

${ }^{1}$ Ashley Akerman*, ${ }^{1} \mathrm{Jim}$ Cotter, ${ }^{2}$ Tord Kjellstrom. ${ }^{1}$ School of Physical Education, Sport and Exercise Sciences; University Of Otago, Dunedin, New Zealand; ${ }^{2}$ National Centre for Epidemiology and Population Health; Australian National University, Canberra, Australia

\subsection{6/OEM-2019-EPI.196}

Recent global analysis of current and future ambient heat conditions has shown that occupational heat exposure in Pacific countries, measured or projected as WBGT, is among the most extreme in the world. Many Pacific countries face a serious and unprecedented collision of adverse environmental, sociocultural/demographic and chronic health factors. Environmentally, high temperatures and humidity exist several months each year, reducing physical work capacity. Conservative assessments reveal annual loss of healthy work hours for a person working at $400 \mathrm{~W}$ metabolic rate in countries like Papua New Guinea, Kiribati, Nauru and Guam increasing from $2 \%-3 \%$ to $9 \%-18 \%$ by 2100 , based on the RCP6.0 pathway for climate change. Socio-culturally, such countries have low average income levels and high reliance on primary industries, so occupational and domestic exposure to ambient heat is chronic and somewhat intractable. Health wise, such countries have among the highest burden of chronic cardiometabolic disease in the world. Diabetes is already prevalent $(20 \%-35 \%)$, as is hypertension. Casual links between heat exposure and cardiovascular health problems is strengthening and these health problems are becoming a major threat to health in Pacific countries. A diet that is increasingly more divergent from traditional foods is one key factor, but increasing heat conditions will contribute, particularly for people working in physically intensive occupations. Obesity is also prevalent, which is relevant because it adds to heat production while concurrently reducing heat loss, thereby impairing work capacity and tolerance of humid heat. Collectively these factors compromise thermal tolerance, reduce functional capacity and contribute to cardiometabolic disease. This paper will analyse the physiological base for the heat effects on cardiovascular disease with particular reference to occupational heat exposure, and estimate the impacts of climate change in the Pacific countries as well as identify solutions to reduce the future impacts.

\section{B.6 OCCUPATIONAL HEAT STRESS DUE TO CLIMATE CHANGE: ESTIMATING FUTURE HEAT WAVE HAZARDS}

${ }^{1,2}$ Matthias Otto* 2,3 Tord Kjellstrom, 1,2 ${ }^{\text {Bruno Lemke. }}{ }^{1}$ Nelson Marlborough Institute of Technology (NMIT), Nelson, New Zealand; ${ }^{2}$ Climate Heat Impact Research Programme (CHIRP), Ruby Bay, New Zealand; ${ }^{3}$ Australian National University (ANU), Canberra, Australia

\subsection{6/OEM-2019-EPI.197}

Exposure to extreme heat negatively affects occupational health. Heat stress indices like Wet Bulb Globe Temperature (WBGT) combine temperature and humidity and allow quantifying the climatic impact on human physiology and clinical health. Multi-day periods of high heat stress (aka. heat waves) affect occupational health and productivity independently from the absolute temperature levels; e.g. well-documented heatwaves in Europe caused disruption, hospitalisations and deaths (2003 French heat wave: more than 1000 extra deaths, 15-65 years, mainly men) even though the temperatures were within the normal range of hotter countries.

Climate change is likely to increase frequency and severity of periods of high heat stress. However, current global gridcell based climate models are not designed to predict heat waves, neither in terms of severity or frequency.

By analysing 37 years of historic daily heat index data from almost 5000 global weather stations and comparing them to widely used grid-cell based climate model outputs over the same period, our research explores methods to assess the frequency and intensity of heat waves as well as the associated occupational health effects at any location around the world in the future.

Weather station temperature extreme values (WBGT) for the 3 hottest days in 30 years exceed the mean WBGT of the hottest month calculated from climate models in the same grid-cell by about 2 degrees in the tropics but by 10 degrees at higher latitudes in temperate climate regions.

Our model based on the relationship between actual recorded periods of elevated heat-stress and grid-cell based climate projections, in combination with population and employment projections, can quantify national and regional productivity loss and health effects with greater certainty than is currently the case. 